\title{
Re-examining the machining frictional boundary conditions using fractals
}

\author{
S Raman* and J Chellaperumal \\ School of Industrial Engineering, University of Oklahoma, Norman, Oklahoma, USA
}

\begin{abstract}
This paper presents experimental evidence for the existence of non-Euclidean contact geometry at the tool-chip interface in the machining of aluminium alloy, which challenges conventional assumptions. The geometry of contact at the tool rake face is modelled using fractals and a dimension is computed for its description. The variation in the fractal dimension with the cutting speed is explored.
\end{abstract}

Keywords: machining frictional boundary, fractals, tool-chip interface, aluminium alloy

\section{INTRODUCTION}

The geometry of several phenomena within machining such as chip fragmentation, friction in the rake face (proportions of sliding and sticking zones), fracture of tools, tool wear and surface roughness appear as fractals at least on some scales. A fundamental non-Euclidean reevaluation of the machining friction using fractals can help to improve the understanding of the process effects such as temperatures, stresses and failure in machining, thus impacting cutting efficiency, tool design, material selection, machining parameter estimation and product quality.

Intuitively, a microscopic evaluation of the tool-chip interface can reveal that pictorial analogies can be drawn to describe it by fractal geometry. The most common models for friction used in force and energy calculations in machining are the Merchant model (pure sliding) and the Zorev [1] model (Fig. 1a). A microscopic evaluation of frictional conditions by Wright et al. [2] suggested that the friction at the toolchip interface is more complex and dynamic than assumed before. They illustrated the existence of relatively large proportions of sliding areas near the cutting edge and at the rear end of contact through experimental analysis. A smaller proportion of seizure islands also existed near the cutting edge and at the rear of the contact. These contradict Zorev's macroscopic frictional model at the interface for explaining the experimental

The MS was received on 28 February 2002 and was accepted after revision for publication on 5 April 2002.

${ }^{*}$ Corresponding author: School of Industrial Engineering, University of Oklahoma, 202 West Boyd, Suite 124, Norman, OK 73019-1022, USA. observations in machining. Consequently, friction must be investigated on a fundamental level, and a generalized model developed for the assumed configurations presented in Figs $1 \mathrm{~b}$ and $\mathrm{c}$.

One reason that researchers have not tried to model the frictional contacts is due to their apparent non-Euclidean nature and the unavailability of mathematical tools to model them. Since 1983, fractal mathematics have been successfully developed to model fragmented patterns in nature. In fact, good success has been reported in the application of fractals in modelling roughness, clustercluster aggregation and fluid fingering that have resulted in improved understanding within these fields. We propose that the fractal approach not only offers us a chance to model the mechanics and dynamics of the tool-chip frictional interface accurately but also provides the scope for developing a unified and generalized model for friction at the interface. The material, geometry and cutting parameters will change the fractal dimension(s), but the general model(s) will remain the same.

\section{FRACTALS AND FRACTAL DIMENSION}

Traditional geometry and classical calculus are used to model and analyse shapes and patterns observed in nature [3]. In order to study shapes that cannot be modelled using conventional tools, a new type of geometry was put forward by Mandelbrot [4] that describes fragmented patterns and irregular sets and leads to a family of shapes called 'fractals'. Every fractal curve has a fractal dimension associated with it, which is different from the topological dimension. Dimension gives rise to two important properties of fractals: self-similarity and self-affinity. In self-similar fractals, every part of 


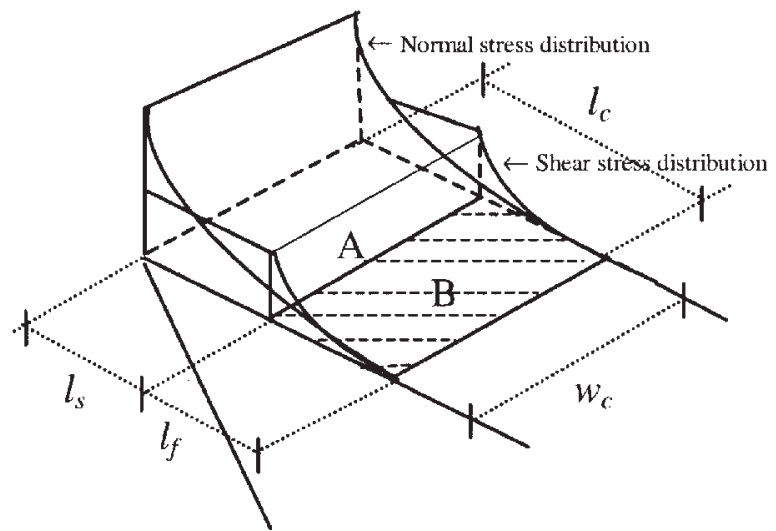

a)

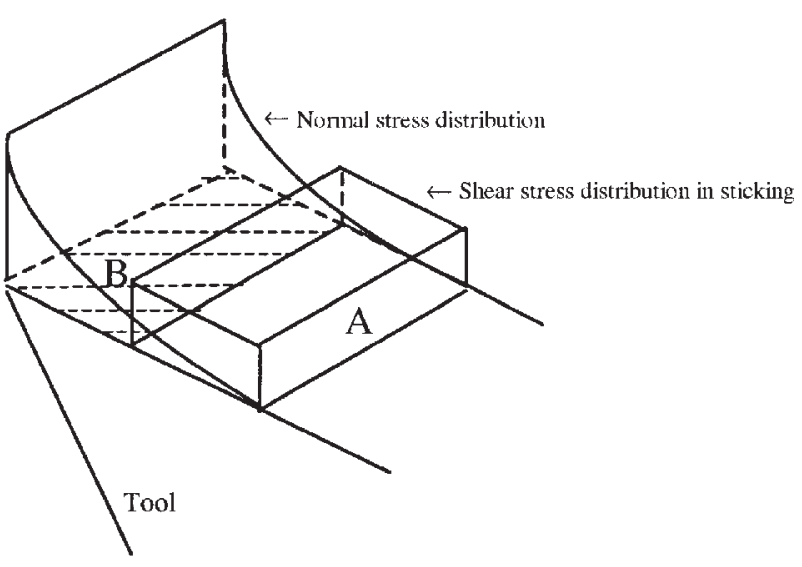

b)

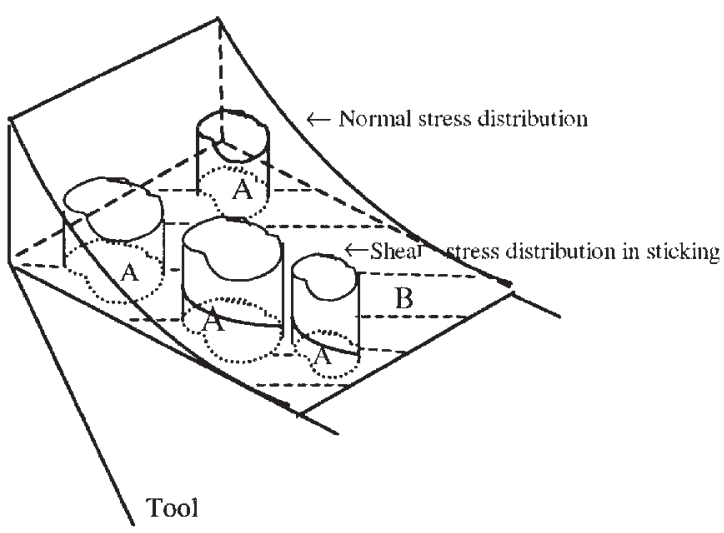

c)

Fig. 1 Hypothetical scenarios of sliding/sticking at the tool rake. Simple orthogonal cutting assumptions are used for developing these scenarios

the fractal looks like the whole with a magnification factor. The surface patterns of objects and wear pattern in the cutting tools are seemingly self-similar. Self-affine fractals are those in which the magnification is not uniform in both the axes.
Recently, there have been numerous applications of fractals in many fields including powder technology, mining, material science, geology and computer vision as surveyed by Kaye [5]. To date, no attempts are evident that quantify friction conditions in machining using fractals. Some efforts $[6,7]$ have been made, however, in the description of general sliding friction as well as surface characterization using fractals or fractal geometry.

In the box-counting technique, grid overlays of various sizes $\lambda$ are used to count the number of boxes $N$ occupied by the profiles or islands whose fractal dimensions are to be determined. The dimension $D$ is given by the slope of the $\log -\log$ plot of $N$ versus $\lambda$. Thus the fractal dimension is given as

$$
D=\lim _{\lambda \rightarrow 0}\left(\frac{\log N}{\log (1 / \lambda)}\right)
$$

In this work, box counting was performed on scanning electron micrographs $(30 \times)$ of the tool rake face using grids of sizes $150,100,75,50,25$ and 15 pixels. The marking and counting of the boxes were carried out with the help of Sigma Scan Pro ${ }^{\mathrm{TM}}$ software.

\section{EXPERIMENTAL VERIFICATION OF FRACTAL BOUNDARY CONDITIONS}

\subsection{Experimental design}

Preliminary experiments conducted on various aluminium alloy and steel grades with carbide and TiN-coated tools were quite encouraging in verifying the fractal nature of the seizure islands. Consequently, detailed experimentation and analysis was conducted. Cutting conditions were selected to allow the study of adhesion resulting from the formation of the built-up edge as well during the formation of a flow zone. As expected, at very low speeds the seizure islands were almost continuous, while at recommended speeds of machining the adhesion was more sporadic. Analyses were based on the post-mortem observations of the rake face. The cutting speed was varied in experiments while machining two grades of aluminium alloy. Other variables varied during the experiments were the rake geometry, tool coating and feed rate. The effect of varying the cutting speed was studied on the computed fractal dimension. The other machining parameters did not exhibit much variation with the fractal dimensions during the preliminary experiments.

The aluminium alloy 6061-T6511 chosen was extruded while the aluminium alloy 2024-T351 was cold rolled. The work pieces were obtained as solid metal billets of 6 in diameter and 36 in length and were prepared for turning experiments. All the experiments were carried out under dry conditions without any coolants. 


\section{RESULTS AND DISCUSSION}

At first glance, rake-face micrographs confirmed the limitations of the Merchant- and Zorev-type assumptions. Visual analysis of the replication tests, with aluminium alloy 2024-T351, revealed similar adhesion patterns, a sliding region surrounded by seizure islands. Consequently, the computed fractal dimensions $D$ using the box-counting method for these cuts were very similar in value. The average value of $D$ for these cuts was $1.6488 \pm 0.0089$. The extent and distribution of the seizure islands were seemingly identical for all cuts. Similar consistency was noted while machining aluminium alloy 6061-T6511 with a carbide tool and the fractal dimensions were determined to be $1.748 \pm 0.006$. The consistency in the values of $D$ for the replicate cuts implies that the fractal dimension $D$ could be a consistent descriptor.

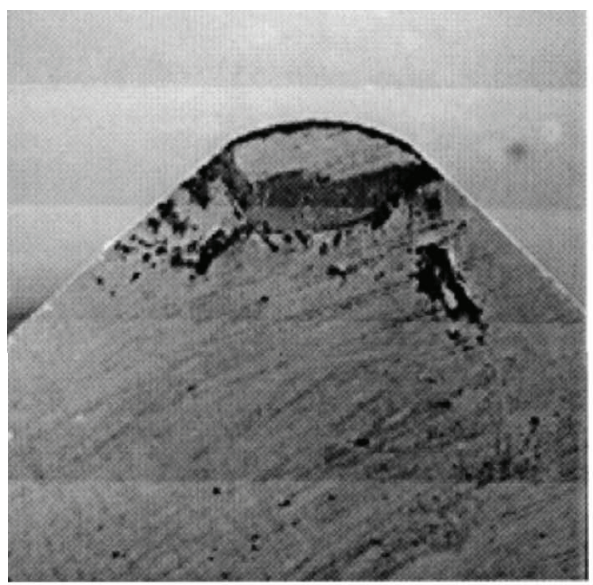

$2024(1175 \mathrm{fpm})$

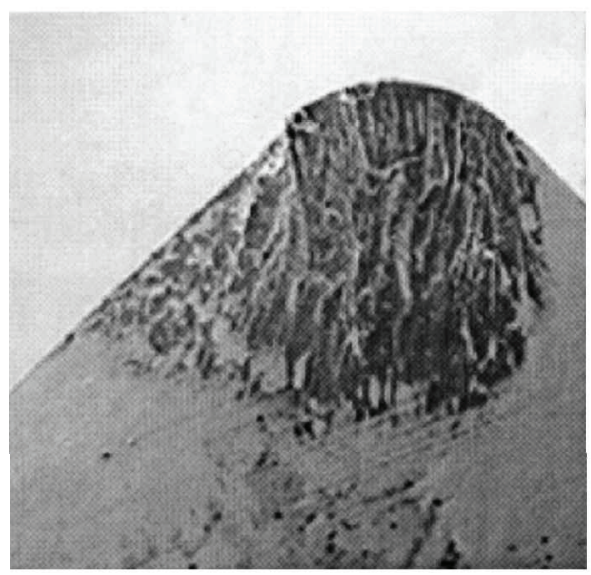

2024 (252 fpm)
It was found that the fractal dimension $D$ increased with a decrease in the cutting speed, in a designed set of cuts (T2). Visual analysis confirmed that the sticking pattern changed with a change in cutting speed. Initially, at the lowest speed, the adhesion pattern is extensive with an area of complete seizure near the tip, extending all the way to the centre of the tool. At the next higher speed, the area of seizure has contracted dramatically, with a clear (adhesion-free) area in the middle. At higher speeds, further contraction of the seizure area takes place in the middle, and some seizure closer to the centre of the tool. At the highest speed, very little adhesion occurs at the every edge of the tool, followed by a clear area and then a band of seizure.

A second set of trials (T3) investigated the possible variation in $D$ with a change in cutting speed at a lower feed rate. This was carried out to investigate the possible effect of feed rate on speed versus $D$. It was

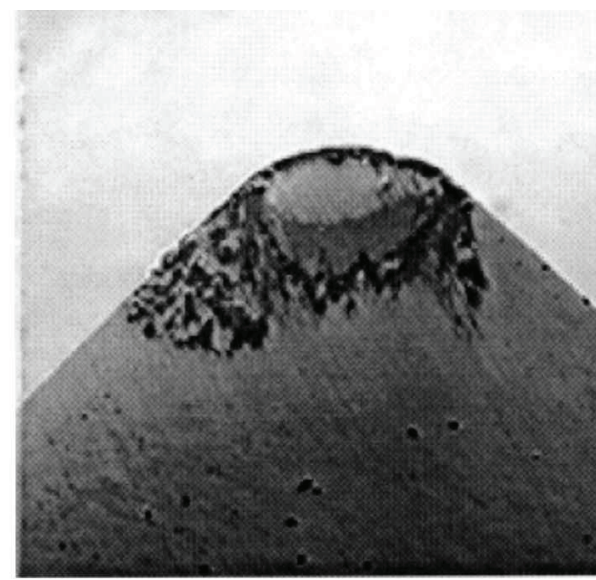

$2024(851 \mathrm{fpm})$

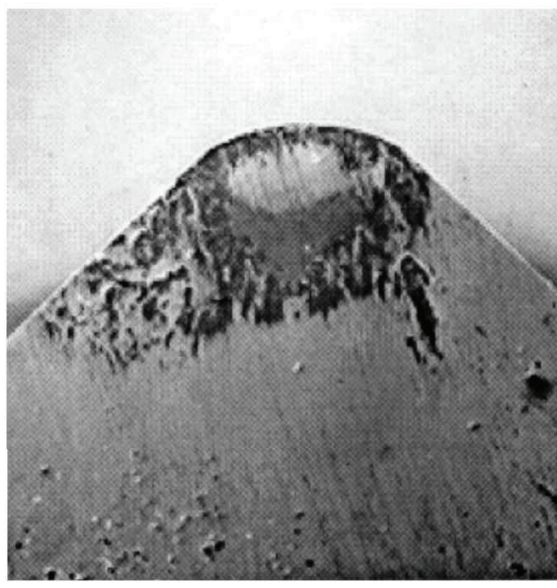

$2024(606 \mathrm{fpm})$

Fig. 2 Friction patterns observed in the turning of aluminium alloy 2024-T351 with an uncoated carbide tool (Tool material, uncoated carbide, negative rake angle SNG422-K68; cutting time (constant), 2.58 min; depth of cut (constant), $0.04 \mathrm{in}$; feed rate (constant), $0.014 \mathrm{in} / \mathrm{rev}$ ) 


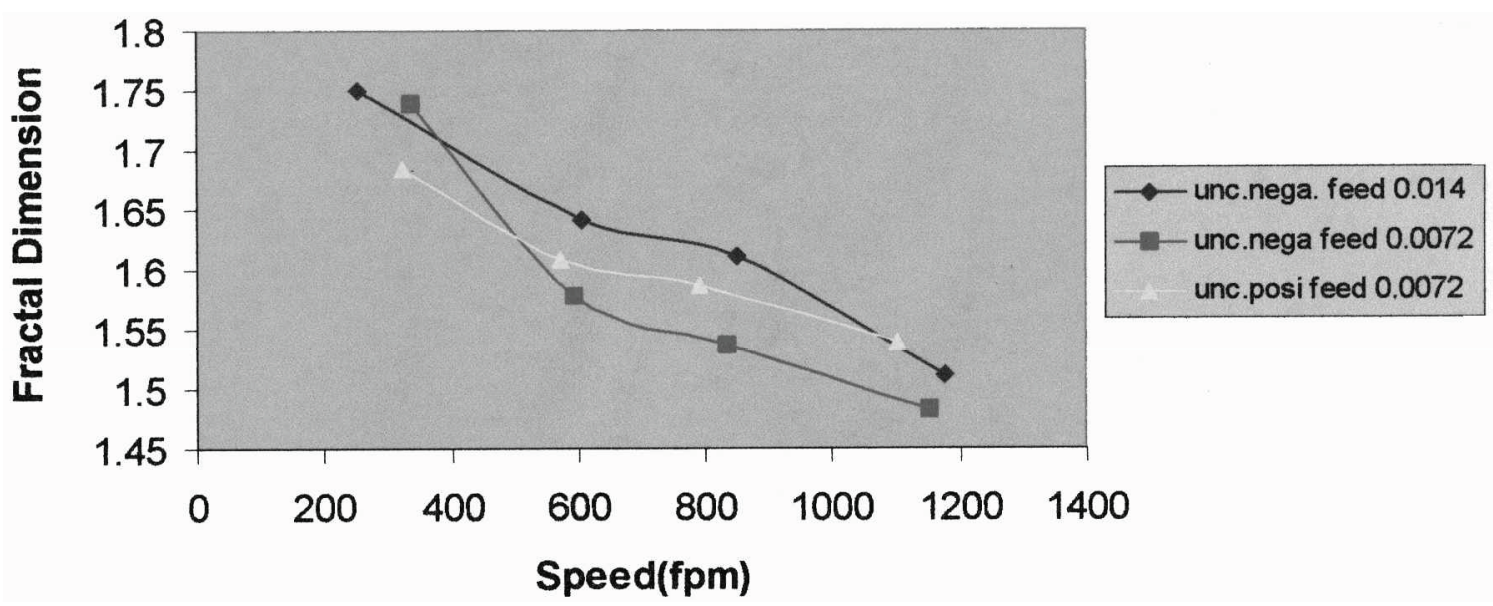

Fig. 3 The variation in the fractal dimension $D$ with the cutting speed

again found that the fractal dimension increased with a decrease in the cutting speed. On comparison, the range of $D$ was $1.51-1.75$ in T2 tests whereas the range was $1.48-1.73$ in $\mathrm{T} 3$ tests. The difference in the range was not noteworthy, although the feed rate was halved.

In trial set $\mathrm{T} 4$, a TiN-coated positive rake angle tool insert of type SPG422 grade (Kennametal ${ }^{\circledR}$ ) K730 was used instead of an uncoated negative rake angle tool insert of type SNG422 grade (Kennametal ${ }^{\circledR}$ ) K68 used in $\mathrm{T} 1$ to $\mathrm{T} 3$ experiments. This was intended to find any possible influence of coating material on the fractal dimension $D$. The cutting speed was varied in all the four cuts. A similar trend of $D$ with speed as before was observed. The range of $D$ was $1.40-1.65$. It could be imagined that the reduction in $D$ compared with the values obtained when using an uncoated tool might be due to the influence of the coating material and/or rake angle in altering the frictional contact and consequently $D$. However, more experiments must be conducted and the influence of cutting time evaluated before concrete conclusions are drawn. Trials T5 were performed using an uncoated positive rake angle tool insert of type SPG422 grade K68 to note the effect of the tool rake angle on the seizure pattern and the fractal dimension $D$. Similar to the other trials the cutting speed was the only variable and the fractal dimension $D$ ranged between 1.54 and 1.68. To determine the effect of the tool geometry on $D$, the values of $D$, under the same cutting conditions at $1175 \mathrm{ft} / \mathrm{min}$, using an uncoated negative rake angle tool and an uncoated positive rake angle tool were compared. The value of $D$ was 1.486 for the former while $D$ was 1.538 for the latter. Thus it was interpreted that the tool geometry could have an effect on the seizure pattern and $D$. Similarly, tests were conducted with aluminium alloy 6061-T6511 and similar observations were noted.

Sample observations for the aluminium alloy 2024-T351 tests are summarized in Fig. 2. The fractal dimension variations with speed at some parameter combinations are shown for aluminium alloy 2024-T351 in Fig. 3. Based on these observations it could be stated that the speed effect on the fractal dimension is also visually revealing. As the speed increases, the seized material band adjacent to the edge becomes thinner, thereby opening up a large sliding island in the middle. Sporadic seizure follows in the rear of the contact zone. These observations, at the very least, pose a challenge to conventional model assumptions regarding seizure and sliding and their domains of existence.

\section{CONCLUDING REMARKS}

This paper is restricted to analysis based on observations rather than statistical analysis that must be conducted to verify our findings fully. More work needs to be done in the re-examination of friction in machining using fractals, through a careful evaluation of literature in all areas.

\section{ACKNOWLEDGEMENTS}

This work was partly supported by the National Science Foundation (NSF) under Exploratory Grant DMII9910219. The views and opinions expressed in this paper are those of the authors and not necessarily those of the NSF.

\section{REFERENCES}

1 Zorev, N. N. Interrelationship between shear processes occurring along tool face and on shear plane in metal cutting. In Proceedings of the International Production Engineering Research Conference, 1963, pp. 42-49.

2 Wright, P. K., Horne, J. G. and Tabor, D. Boundary conditions at the chip-tool interface in machining: comparison between seizure and sliding friction. Wear, 1979, 54, 371-390. 
3 Falconer, K. Fractal Geometry, 1990 (John Wiley, New York).

4 Mandelbrot, B. Fractal Geometry of Nature, 1983, pp. 1-30 (Freeman, San Francisco, California).

5 Kaye, B. H. Randomwalk Through Fractal Dimensions, 1989, pp. 1-80 (VCH, New York).
6 Majumdar, A. and Bhushan, B. Role of fractal geometry in roughness characterization and contact mechanics of surfaces. Trans. ASME, J. Tribology, 1990, 112, 205-216.

7 Majumdar, A. and Bhushan, B. Fractal model of elasticplastic contact between rough surfaces. Trans. ASME, J. Tribology, 1991, 113, 1-11. 